\title{
ABBREVIATING PROOFS BY ADDING NEW AXIOMS
}

\author{
BY ANDRZEJ EHRENFEUCHT AND JAN MYCIELSKI
}

Communicated by Dana Scott, July 9, 1970

The purpose of this note is to state precisely and prove the following informal statement: If $T$ is a theory and $\alpha$ is a new axiom such that $T+\operatorname{non} \alpha$ is an undecidable theory then some theorems of $T$ have much shorter proofs in $T+\alpha$ than in $T$. Notice that if $T$ is an essentially undecidable theory, like e.g. arithmetic, this conclusion will be true provided $\alpha$ is a sentence which is not a theorem of $T$, since then $T+$ non $\alpha$ is undecidable.

Let $T$ be a formalized theory which among its logical functors has the negation 7 , the implication $\rightarrow$, and the alternative $\vee$. Let $\sigma$ and $\tau$ be variables ranging over sentences formulated in the language of $T$ and $\alpha$ one fixed such sentence. We denote by $\Gamma^{\top}{ }^{\top}$ the Gödel number of $\sigma$, although here $\Gamma T$ is just any one-to-one map of the set of sentences into the set of positive integers. For any theorem $\tau$ of $T$ let $W(\tau)$ be also a positive integer measuring in some way the length of the shortest proof of $\tau$ in $T$. But all we need about ${ }^{\top} T$ and $W$ are the following conditions:

(i) The set $\left\{2^{n}\left(2\left\ulcorner\tau^{\top}+1\right): \tau\right.\right.$ is valid in $T$ and $\left.W(\tau) \leqq n\right\}$ is recursive.

(ii) There are recursive functions $g$ and $h$ such that for every $\sigma$

$$
W(\alpha \rightarrow(\alpha \bigvee \sigma)) \leqq g(\ulcorner\sigma\urcorner), \quad h(\ulcorner\sigma\urcorner)=\Gamma_{\alpha} \bigvee \sigma^{\top}
$$

The meaning of (i) is that there is an algorithm to check if $\tau$ has a proof of length $\leqq n$. This stipulation entails that the set of Gödel numbers of the theorems of $T$ is recursively enumerable. It is clear that reasonable $\Gamma 7$ and $W$ satisfy (i) and (ii).

LEmma. If the theory $T+\neg \alpha$ is undecidable, i.e. the set $\left\{\Gamma^{\top}: \alpha \vee \sigma\right.$ is valid in $T\}$ is not recursive, then there is no recursive function $f$ such that

$$
W(\tau) \leqq f(W(\alpha \rightarrow \tau))
$$

for every $\tau$ valid in $T$.

Proof. Suppose to the contrary that (1) holds. We can assume without loss of generality that $f$ is nondecreasing. Then by (1) and (ii) we get

AMS 1970 subject classifications. Primary 02G05, 02F27.

Key words and phrases. Proofs, axioms, length, recursive sets.

Copyright @ 1971, American Mathematical Society 


$$
W(\alpha \bigvee \sigma) \leqq f(W(\alpha \rightarrow(\alpha \bigvee \sigma))) \leqq f\left(g\left(\left\ulcorner\sigma^{\top}\right)\right)\right.
$$

Hence if we want to check for a given positive integer $k$ if $k \in\left\{\Gamma^{\top}\right.$ : $\alpha \bigvee \sigma$ is valid in $T\}$ it is enough to evaluate $f(g(k)), h(k)$ and check if $2^{f(g(k))}(2 h(k)+1) \in\left\{2^{n}(2\ulcorner\tau\urcorner+1): \tau\right.$ is valid in $T$ and $\left.W(\tau) \leqq n\right\}$.

By (i) this constitutes a decision procedure, contrary to the supposition of the Lemma. Q.E.D.

To apply this Lemma to the theory $T+\alpha$ we must assume that the function $W^{*}(\tau)$ measuring the length of the shortest proof of $\tau$ in $T+\alpha$ is such that

(iii) There exists a recursive function $r$ such that

$$
W^{*}(\tau) \leqq r(W(\alpha \rightarrow \tau))
$$

for every $\tau$ valid in $T$.

This again is true for any $\alpha$ and most reasonable $W$ and $W^{*}$ we can think of.

THEOREM. If the theory $T+7 \alpha$ is undecidable then there is no recursive function s such that

$$
W(\tau) \leqq s\left(W^{*}(\tau)\right)
$$

for every theorem $\tau$ of $T$.

Proof. Suppose to the contrary that (2) holds. We can assume without loss of generality that $s$ is nondecreasing. Then by (2) and (iii) we get

$$
W(\tau) \leqq s(r(W(\alpha \rightarrow \tau))),
$$

which contradicts our Lemma. Q.E.D.

Note AdDed on October 25, 1970. See M. A. Arbib, Theories of abstract automata, Prentice-Hall, Inc. 1969, Chapter 7.4, pp. 261267, for related results and references.

University of Southern California, Los Angeles, California 90007

University of Colorado, Boulder, Colorado 80302 